\title{
Exact analysis of surface field reduction due to field-emitted vacuum space charge, in parallel-plane geometry, using simple dimensionless equations
}

\author{
Richard G. Forbes ${ }^{\text {a) }}$ \\ Advanced Technology Institute (X1), Faculty of Engineering and Physical Sciences, University of Surrey, \\ Guildford, Surrey GU2 7XH, United Kingdom
}

(Received 8 May 2008; accepted 20 August 2008; published online 20 October 2008)

\begin{abstract}
This paper reports (a) a simple dimensionless equation relating to field-emitted vacuum space charge (FEVSC) in parallel-plane geometry, namely $9 \zeta^{2} \theta^{2}-3 \theta-4 \zeta+3=0$, where $\zeta$ is the FEVSC "strength" and $\theta$ is the reduction in emitter surface field ( $\theta=$ field-with/field-without FEVSC), and (b) the formula $j=9 \theta^{2} \zeta / 4$, where $j$ is the ratio of emitted current density $J_{P}$ to that predicted by Child's law. These equations apply to any charged particle, positive or negative, emitted with near-zero kinetic energy. They yield existing and additional basic formulas in planar FEVSC theory. The first equation also yields the well-known cubic equation describing the relationship between $J_{P}$ and applied voltage; a method of analytical solution is described. Illustrative FEVSC effects in a liquid metal ion source and in field electron emission are discussed. For Fowler-Nordheim plots, a "turn-over" effect is predicted in the high FEVSC limit. The higher the voltage-to-local-field conversion factor for the emitter concerned, then the higher is the field at which turn over occurs. Past experiments have not found complete turn over; possible reasons are noted. For real field emitters, planar theory is a worst-case limit; however, adjusting $\zeta$ on the basis of Monte Carlo calculations might yield formulae adequate for real situations. (C) 2008 American Institute of Physics. [DOI: 10.1063/1.2996005]
\end{abstract}

\section{INTRODUCTION}

Charged particles emitted into vacuum, and drawn by an applied voltage toward an "extractor" electrode, constitute a "vacuum space charge." If large enough, this space charge reduces the emitter surface field and limits the emission current for given applied voltage. This can occur with both thermally and field-induced emissions, but differences in detail make it best to treat these separately. In this paper, the terms "emission" and "field emission" cover both electron and ion emission.

The theory of thermally emitted vacuum space charge derives from the papers of Child ${ }^{1}$ and Langmuir. ${ }^{2,3}$ Although Jaffé's ${ }^{4} 1920$ analysis is relevant, the theory of field-emitted vacuum space charge (FEVSC) properly begins with the 1929 paper of Stern et al., ${ }^{5}$ which followed up the wellknown work of Fowler and Nordheim ${ }^{6}$ (FN) on the basic theory of cold field electron emission (CFE). Subsequently, Cockburn, ${ }^{7}$ Ivey, ${ }^{8}$ Barbour et al., ${ }^{9}$ and many others (including Refs. 10-36), developed relevant theory and applied it to specific problems. Treatments of FEVSC, based on applying Monte Carlo methods to the emission process, are embedded in commercial and other computer programs ${ }^{37-40}$ used to analyze the optical properties of electron and ion systems.

This paper aims to make FEVSC effects (and the associated mathematics) easier to understand by presenting an alternative mathematical treatment of the basic onedimensional (1D) planar-geometry FEVSC model. It involves a dimensionless biquadratic equation, Eqs. (14a) and

${ }^{\text {a)} E l e c t r o n i c ~ m a i l: ~ r . f o r b e s @ i e e e . o r g . ~}$ (14b) below, that I call the dimensionless planar FEVSC equation. This differs from other reported dimensionless FEVSC equations. ${ }^{7,8,15,26,30,33}$ Equations (14a) and (14b) have an exact solution for the field reduction factor $\theta$ in terms of the FEVSC "strength" $\zeta$ (see definitions below). The main empirical parameters involved $\left(V, F_{P}, F_{L}, J_{P}\right.$, and $J_{L}$ below) have definitions that make them positive for both positive and negative particle emissions. The theory applies to any form of field-induced charged-particle emission, provided that all particles are the same and have, at emission, a narrow range of kinetic energy near zero.

In technology, FEVSC effects can help (as when they stabilize an emission current), or hinder (as when they inhibit sharp focusing of particle beams). Contexts where improved FEVSC theory may be useful include the liquid metal ion source (LMIS) ${ }^{36,41}$ (used in focused ion beam machines ${ }^{42}$ and the field emission electric propulsion of spacecraft ${ }^{43}$ ), Spindt arrays, ${ }^{27,28,44}$ and the proposed use of carbon nanotubes as bright field electron sources (e.g., Ref. 45). There also remains an older problem with field electron emitters. At high emission current densities, small departures are observed $^{9}$ from the behavior predicted by the standard FNtype emission model; ${ }^{46,47}$ both model breakdown and FEVSC effects have been suggested as causes, ${ }^{48,49}$ but the true explanation is still not firmly decided. Other contexts are work on multidimensional space-charge-limited flow (e.g., Ref. 50), on FEVSC in nanogaps (e.g., Ref. 51), and on FEVSC effects in charged-particle sources, optical systems, and other complicated geometries, such as accelerators (e.g., Ref. 52).

In general, field emission sources have pointlike geom- 
etry. A 1D planar-geometry model has obvious limitations, but also advantages, in that it can be treated analytically and has clear numerical predictions. These provide a lower bound ("worst case scenario") for the predicted onset of FEVSC effects, even for nonplanar geometries.

To deal correctly with FEVSC effects in real geometries, very complex models are needed that often involve extensive computation. When developing such models and interpreting outputs, better understanding of the basic 1D planar model might be helpful. As suggested below, it might be possible to adjust its solution empirically, for use as an approximation applicable to specified nonplanar geometries.

The 1D planar model uses a classical-continuum approach to replace the real behavior of emitted particles by the classical behavior of their time-average charge distribution. This will be adequate for some aspects of source behavior, in particular, time-average current/voltage characteristics and the average FEVSC-induced surface field reduction. However the model cannot describe aspects determined by stochastic Coulomb interactions ${ }^{34}$ between individual emitted particles (namely, energy-distribution broadening and trajectory displacement). Also, the model is not exact for particles accelerated to relativistic velocities. ${ }^{16}$

The paper's structure is as follows. Sec. II derives the dimensionless planar FEVSC equation and its exact solutions. Sec. III discusses approximate solutions. Sec. IV shows how the usual cubic equation, ${ }^{9}$ linking current density, and applied voltage, relates to the derived equation. Sec. V presents some illustrative applications. Sec. VI comments on the possibility of developing an approximate form of solution for nonplanar geometry. Sec. VII notes limitations due to relativistic mass increase. Sec. VIII provides a summary. Appendix 1 notes mathematical features of the analytical solution of cubic equations.

Aspects of these results have been reported in outline elsewhere. $^{53}$

\section{THEORETICAL DERIVATIONS}

\section{A. Definitions and conventions}

In this paper, $e$ denotes the elementary positive charge, $n e$ the magnitude of the charge on the emitted particle, $m$ its mass, and $\varepsilon_{0}$ the electric constant. The emission geometry is a pair of plane-parallel plates (the "emitter" and the "extractor") a distance $x_{D}$ apart, with a voltage of magnitude $V$ between them. Distance from the emitter's surface (defined as the plane where particles emerge with zero kinetic energy) is denoted by $x$. The field magnitude at this surface is denoted by $F_{P}$, and a field-induced emission process creates an emission current density of magnitude $J_{P}$. All of $n, V, F_{P}$, and $J_{P}$ are thus positive, for both positively and negatively charged particles.

Strictly, the symbol $V$ represents the magnitude, not of the applied voltage difference, but of the classical electrostatic potential difference between the surfaces of the extractor and emitter. If a contact potential difference exists due to work function differences, then the difference in classical electrostatic potential may differ from that in applied voltage by up to a few volts. In FEVSC theory, this distinction be- tween electrostatic potential and voltage may nearly always be disregarded; so this paper follows the literature in referring to $V$ as the "applied voltage."

Following Mair's ${ }^{19}$ terminology, the magnitude $F_{P}$ of the real emitter surface field (whether significant space charge is present or not) is called the "Poisson surface field," and the magnitude $J_{P}$ of the corresponding real emission current density is called the "Poisson emission current density." For the same system geometry ${ }^{54}$ and applied voltage, the emitter surface field that would exist in the absence of emission (and hence the absence of space charge) is called the "Laplace surface field" and is denoted by $F_{L}$. The emission current density that would correspond to a surface field of magnitude $F_{L}$ is called the "Laplace emission current density" and denoted by $J_{L}$.

The "field reduction factor" $\theta$ is defined by

$$
\theta=F_{P} / F_{L}
$$

As shown below, the parameter $\theta$ is a function of the spacecharge strength $\zeta$. For a given system geometry, a space charge of high strength greatly reduces $F_{P}$ below the Laplace value $F_{L}$

In classical electrostatics, the relationship between $F_{L}$ and $V$ is determined by system geometry and can be written as

$$
F_{L}=\beta_{L} V,
$$

where (following the convention of Barbour et al. ${ }^{9}$ ) the symbol $\beta$ denotes voltage-to-surface-field conversion factor. Here, $\beta_{L}$ denotes its Laplace value, i.e., its value in the absence of space charge (thus our " $\beta_{L}$ " is the same as the " $\beta$ " of Barbour et al. ${ }^{9}$ ). In the presence of emission and space charge, a "Poisson conversion factor" $\beta_{P}$ is defined by

$$
F_{P}=\beta_{P} V
$$

For given system geometry, $\beta_{L}$ is independent of applied voltage, provided that no voltage-dependent field penetration or similar effects occur, and that the emitter work function is uniform (so no patch fields ${ }^{55}$ are present). However, $\beta_{P}$ depends on applied voltage and will be significantly less than $\beta_{L}$ when the space-charge strength is high, because $\beta_{P}$ $=\theta \beta_{L}$.

Obviously, for a system geometry consisting of a classically smooth flat planar emitter and a parallel planar extractor, both of infinite extent, with the emitter having uniform work function and no voltage-dependent field penetration, $\beta_{L}=1 / x_{D}$.

For the arguments of logarithms, the standard convention $^{56}$ is used that enclosing a quantity in curly brackets means "measure the quantity in brackets in specified units, and take its numerical value." To avoid ambiguity, the arguments of logarithms (and also of current densities and $\beta$-values) are always expressed in SI base units. Also, the unit "Neper" $(\mathrm{Np})$ is used to measure the difference in values of natural logarithms. (That is, if $\ln \{X / Y\}=1$, then $\ln \{X\}$ is greater than $\ln \{Y\}$ by $1 \mathrm{~Np})$.

Note that attaching the label "Poisson" and the subscript " $P$ " to the real emission quantities is a device intended to 
clarify discussion here. In the literature, these real quantities are usually denoted by the corresponding main symbols, without subscripts.

\section{B. Planar space-charge equation}

To describe the electrostatic effects of space-charge, Child $^{1}$ (and also Langmuir ${ }^{2}$ ) derived, from Poisson's equation, a basic 1D potential equation. In the rationalized meterkilogram-second equation system, this takes the form

$$
d^{2} U / d x^{2}=\left(\kappa J_{P}\right) U^{-1 / 2},
$$

where $U(x)$ is the magnitude of the classical potential difference between point $x$ and the emitter surface at $x=0$, and $\kappa$ is a parameter characteristic of the emitted particle, given by

$$
\kappa=\varepsilon_{0}^{-1}(m / 2 n e)^{1 / 2} .
$$

As noted earlier, these equations apply to particles with any charge, positive or negative, but all must have the same charge. Typical values are $\kappa \cong 1.904 \times 10^{5} \mathrm{~A} \mathrm{~V}^{3 / 2}$ for an electron and $\kappa \cong 6.789 \times 10^{7} \mathrm{~A} \mathrm{~V}^{3 / 2}$ for a $\mathrm{Ga}^{+}$ion.

From Eq. (4), a planar FEVSC equation has been derived, in various different forms, on many occasions. For completeness here, we outline a derivation leading to the Barbour et al. ${ }^{9}$ form. On multiplying Eq. (4) by $2 d U / d x$, it may be integrated directly to give

$$
(d U / d x)^{2}=4\left(\kappa J_{P}\right) U^{1 / 2}+\text { constant. }
$$

At $x=0, U=0$, and $d U / d x=F_{P}$, so Eq. (6) becomes

$$
d x=\left[4 \kappa J_{P} U^{1 / 2}+F_{P}^{2}\right]^{-1 / 2} d U .
$$

This can be integrated by substituting $w=\left(F_{P}^{2}+4 \kappa J_{P} U^{1 / 2}\right)$, which leads to

$$
\begin{aligned}
x+\text { constant }= & \left(1 / 6 \kappa^{2} J_{P}^{2}\right)\left(4 \kappa J_{P} U^{1 / 2}+F_{P}^{2}\right)^{1 / 2}\left(2 \kappa J_{P} U^{1 / 2}\right. \\
& \left.-F_{P}^{2}\right) .
\end{aligned}
$$

$U=0$ when $x=0$, so the constant is $-F_{P}^{3} / 6 \kappa^{2} J_{P}^{2}$. Further, $U=V$ when $x=x_{D}$, so we get the result in the Barbour et al. ${ }^{9}$ form (their $k$ is our $\kappa$ ),

$$
6 \kappa^{2} J_{P}^{2} x_{D}-F_{P}^{3}=\left(4 \kappa J_{P} V^{1 / 2}+F_{P}^{2}\right)^{1 / 2}\left(2 \kappa J_{P} V^{1 / 2}-F_{P}^{2}\right) .
$$

As noted earlier, the derivation of Eq. (4) assumes that particles are emitted normal to the emitting surface with effectively zero kinetic energy. ${ }^{1,2}$ In FEVSC, the surface field $F_{P}$ has to be relatively high for emission to occur, whatever form of charged-particle emission is concerned, so the particle is always removed from the surface quickly. Hence, the details of the distribution of initial kinetic energies are expected to have little influence on space-charge effects. This contrasts with the situation for thermally emitted vacuum space charge, where the surface field is close to zero, and the distribution of initial kinetic energy is important in detailed theory. ${ }^{3}$ Wheeler's work ${ }^{15}$ confirmed that the initial kineticenergy distribution is not important in FEVSC theory, except possibly for low-work-function materials at very high emission currents.

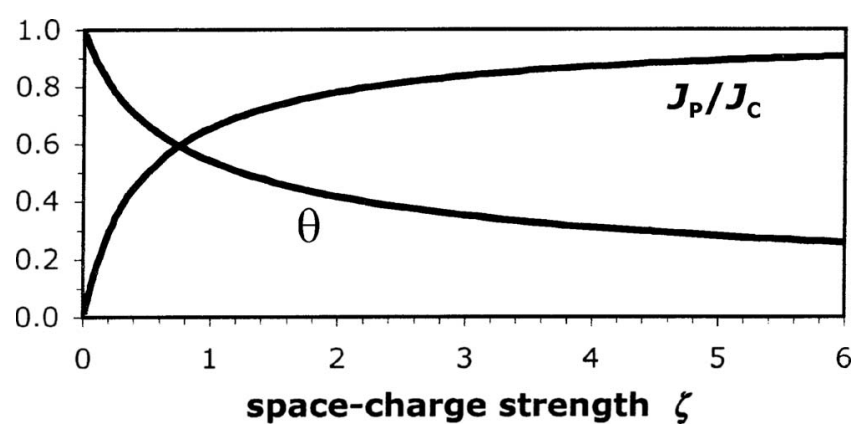

FIG. 1. To show how the field reduction factor $\theta$ and the emission current ratio $J_{P} / J_{C}$ vary with the space-charge strength $\zeta$.

\section{Derivation of a dimensionless equation}

A simpler form, using only dimensionless parameters, can be obtained from Eq. (9). Divide both sides by $F_{P}^{3}$, and use $x_{D}=V / F_{L}=\left(V / F_{P}\right)\left(F_{P} / F_{L}\right)=\theta V / F_{P}$ to give

$$
\begin{aligned}
6 \theta \kappa^{2} V J_{P}^{2} / F_{P}^{4}-1= & \left(4 \kappa V^{1 / 2} J_{P} / F_{P}^{2}+1\right)^{1 / 2}\left(2 \kappa V^{1 / 2} J_{P} / F_{P}^{2}\right. \\
& -1)
\end{aligned}
$$

On defining

$$
\zeta \equiv \kappa V^{1 / 2} J_{P} / F_{P}^{2},
$$

we obtain

$$
\left(6 \theta \zeta^{2}-1\right)=(4 \zeta+1)^{1 / 2}(2 \zeta-1) .
$$

Squaring and simplifying yields

$$
9 \zeta^{2} \theta^{2}-3 \theta-4 \zeta+3=0 .
$$

This equation, called here the dimensionless planar FEVSC equation, has not been previously reported in reviewed literature. It relates the field reduction factor $\theta$ to a dimensionless parameter $\zeta$ that quantifies the space-charge strength. Ivey's parameter ${ }^{8} u$ is related to $\zeta$ by $u=1 / 4 \zeta$.

The factor $J_{P} / F_{p}^{2}$ in $\zeta$ makes it easy to use FN-type equations to calculate $\zeta$ for electrons emitted by CFE. The biquadratic form of Eqs. (14a) and (14b) makes it simple to obtain analytical solutions for either $\theta(\zeta)$ or $\zeta(\theta)$, though often $\theta(\zeta)$ is of more interest.

\section{General solutions}

Clearly the general solution for $\theta(\zeta)$ can be written as

$$
\begin{aligned}
& \theta(\zeta)=\left(1 / 6 \zeta^{2}\right)(1 \pm \sqrt{ } S), \quad \text { where } \\
& S \equiv 1+4 \zeta^{2}(4 \zeta-3) .
\end{aligned}
$$

Formula (14a) has a mathematical branch point at $\zeta=\frac{1}{2}$, at which $S=0$ and $\theta=\frac{2}{3}$. For $\zeta<\frac{1}{2}$ the negative sign must be taken in Eq. (14a), for $\zeta>\frac{1}{2}$ the positive sign. However, $\theta(\zeta)$ is a smoothly varying (monotonically decreasing) function over the whole range of $\zeta$, as shown in Fig. 1.

Similarly, the general solution for $\zeta(\theta)$ is

$$
\zeta(\theta)=\left(1 / 9 \theta^{2}\right)\left\{2 \pm \sqrt{ }\left[4-27 \theta^{2}(1-\theta)\right]\right\},
$$

where for $\theta<\frac{2}{3}$ the negative sign is taken, and for $\theta>\frac{2}{3}$ the positive sign is taken. 


\section{MATHEMATICAL SPACE-CHARGE REGIMES}

Although Eqs. (14a) and (14b) can always be used to generate $\theta$-values from $\zeta$-values, it is instructive to identify "mathematical space-charge regimes" where simpler approximations apply. In terms of increasing $\zeta$-values (decreasing $\theta$-values) these are as below. For the limiting regimes it is easier to work directly from Eq. (13), rather than by approximating Eqs. (14a) and (14b).

\section{A. Negligible-space-charge regime}

When $J_{P}$ (and hence $\zeta$ ) is very small, the terms in $\zeta$ may be neglected in Eq. (13), and the solution is $\theta \approx 1$. That is, $F_{P} \approx F_{L}=\beta_{L} V$, there is negligible surface field reduction due to FEVSC, and the emission current density $J_{P}$, as a function of applied voltage $V$, has the dependence expected from the emission equation and system geometry.

\section{B. Small-space-charge regime}

When $\zeta$ is small but no longer negligible, the term in $\zeta^{2}$ in Eq. (13) can be neglected, giving

$$
\theta \approx 1-\frac{4}{3} \zeta=1-\frac{4}{3} \kappa V^{1 / 2} J_{P} / F_{p}^{2} .
$$

This formula was first obtained by Stern et al., ${ }^{5}$ and was used in their criterion for the absence of significant space-charge effects in CFE. If $\zeta$ is sufficiently small, then Eq. (16) can also be put in the form (in effect) used by Mair: ${ }^{20}$

$$
\theta^{2} \approx 1-\frac{8}{3} \zeta=1-\frac{8}{3} \kappa V^{1 / 2} J_{P} / F_{p}^{2} .
$$

In the part of the range between $\zeta=0$ and $\zeta=\frac{1}{2}$ where $(1-S)$ is sufficiently small, a binomial expansion of $[1+(S$ -1)] may be used to approximate $\sqrt{ } S$ in Eq. (13) as $\left[1+\frac{1}{2}(S-1)-\frac{1}{8}(S-1)^{2}\right]$. This yields an approximation equivalent to Eq. (13) in Stern et al., ${ }^{5}$ namely,

$$
\theta \approx 1-\frac{4}{3} \zeta+3 \zeta^{2}-8 \zeta^{3} .
$$

\section{Branch-point neighborhood}

At the branch point $\zeta=\frac{1}{2}, S$ has a minimum of value zero, and $\theta=1 /\left(6 \zeta^{2}\right)=\frac{2}{3}$. Above $\zeta=\frac{1}{2}, S$ becomes larger rapidly; below $\zeta=\frac{1}{2}, S$ increases relatively slowly to a mathematical maximum of 1 at $\zeta=0$. Near the branch point, there is a narrow range where the best linear approximation is

$$
\theta \approx\left(\frac{2}{3}\right)\left[1+(-4 \pm 2 \sqrt{ } 3)\left(\zeta-\frac{1}{2}\right)\right] .
$$

For $\zeta>\frac{1}{2}$ the positive sign is taken, for $\zeta<\frac{1}{2}$ the negative sign. However, this approximation seems to be of limited practical use, and it may be better to use the general formula.

On either side of this narrow branch-point neighborhood there are intermediate regimes where no useful simple approximation exists and the general solution should be used.

\section{Child's law regime}

When $J_{P}$ is large, the terms in $\zeta$ may be assumed to dominate in Eq. (12), and the outcome is a dimensionless form of Child's law, ${ }^{1,57}$ namely,

$$
\theta \approx \frac{2}{3} \zeta^{-1 / 2} .
$$

If Eqs. (1), (2), and (11) are used to replace $\theta$ and $\zeta$, we obtain the more familiar forms of Child's law, which gives the fully space-charge-limited current density $J_{C}$ as

$$
J_{C}=\left(\frac{4}{9}\right) \kappa^{-1} V^{3 / 2} / x_{D}^{2}=\left(\frac{4}{9}\right) \kappa^{-1} \beta_{L}^{2} V^{3 / 2}=\left(\frac{4}{9}\right) \kappa^{-1} \beta_{L}^{1 / 2} F_{L}^{3 / 2} .
$$

In Eq. (14b), when $\zeta$ is large we have $S \sim 16 \zeta^{3}$, so both $S$ and $+{ }_{\sqrt{ }} S$ become large in comparison with unity. In such circumstances, Eq. (14a) reduces to

$$
\theta \approx \frac{2}{3} \zeta^{-1 / 2}[1-3 /(4 \zeta)]^{1 / 2} .
$$

Clearly, Eq. (22) has the form of the Child's law result, multiplied by a correction factor that gets closer to unity as $\zeta$ gets larger.

\section{E. Partial space-charge equations}

From the definitions of $\theta$ and $\zeta$, it can be shown that

$$
9 \theta^{2} \zeta / 4=J_{P} / J_{C} \equiv j,
$$

where $j$ is defined by Eq. (23). Multiplying both sides of Eq. (15) by $\left(9 \theta^{2} / 4\right)$ yields

$$
J_{P} / J_{C}=\left(\frac{1}{2}\right)\left\{1 \pm \sqrt{ }\left[1-\left(\frac{27}{4}\right) \theta^{2}(1-\theta)\right]\right\} .
$$

This is the dimensionless fomula derived by Ivey ${ }^{8}$ as his Eq. (15), and plotted as his Fig. 1.

Similarly, squaring Eqs. (14a) and (14b) and then multiplying by $9 \zeta / 4$ yields

$$
J_{P} / J_{C}=\left(1 / 16 \zeta^{3}\right)(1+S \pm 2 \sqrt{ } S),
$$

where $S$ is given by Eq. (14b), and the negative sign is taken if $\zeta<\frac{1}{2}$, the positive sign if $\zeta>\frac{1}{2}$. This equation has not previously been reported. Figure 1 here shows $J_{P} / J_{C}$ as a function of $\zeta$, for $0 \leq \zeta \leq 6$.

Using Eq. (23) to substitute $\zeta=\left(\frac{4}{9}\right) j \theta^{-2}$ into Eq. (13) yields

$$
j^{2}-j+(27 / 16) \theta^{2}(1-\theta)=0 .
$$

This is the dimensionless equation derived by Gonçales et $a l .{ }^{33}$ Eqs. (23)-(26) are most useful in so-called "partial space-charge conditions."

\section{F. Discussion}

This analysis shows that all the usual simple 1D planar FEVSC formulas, and some additional ones, can be deduced from the dimensionless Eq. (13), its exact analytical solutions Eqs. (14a), (14b), and (15), and the relation (23) that links the three dimensionless quantities $\zeta, \theta$, and $j$. These equations serve to integrate 1D planar FEVSC theory.

Equation (13) has two terms that dominate at low $\zeta$-values and a different two that dominate at high $\zeta$-values. This mathematical structure, also present in Eq. (27) below, ensures that, physically, the system moves smoothly from the low- $\zeta$ situation, where the $J_{P}(V)$ characteristic is given by a formula specific to the form of emission being discussed, to the high- $\zeta$ situation, where the $J_{P}(V)$ characteristic obeys Child's law, irrespective of emission mechanism. 
Note that the $J_{P}\left(F_{P}\right)$ relation is, at all applied voltages, that for the emission mechanism being discussed; FEVSC effects cause no breakdown in this $J_{P}\left(F_{P}\right)$ relationship. What happens is that, as $\zeta$ increases, the Poisson conversion factor $\beta_{P}=F_{P} / V$ changes from being nearly constant to being a function of applied voltage and surface field. The observed $i(V)$ relationship gives the impression of breakdown in the $J_{P}\left(F_{P}\right)$ relationship only if it is not realized that $\beta_{P}$ is no longer constant.

\section{RELATIONSHIP BETWEEN $\boldsymbol{J}_{P}$ AND $\boldsymbol{V}$}

As just shown, formulas (14a) and (14b) are useful for core theoretical purposes, in particular for identifying FEVSC regimes. In the high-FEVSC limit, they provide the Child's law prediction for $J_{P}(V)$. However, to predict $J_{P}(V)$ in other circumstances, a specific emission equation must be provided for $J_{P}\left(F_{P}\right)$. To prepare, we reinsert Eqs. (1), (2), and (11) into Eq. (13), and multiply through by $V^{-1 / 2}$ to obtain

$$
G_{c}(V) \equiv\left(c_{3 C}+c_{3 L}\right) V^{-3 / 2}+c_{1 L} V^{-1 / 2}+c_{0 C}=0,
$$

where the coefficients are given by $c_{3 C}=9\left(\kappa \beta_{L}^{-1} F_{P}\right)^{2}\left(J_{P} / F_{P}^{2}\right)^{2}, \quad c_{3 L}=-3 \beta_{L}^{-1} F_{P}, \quad c_{1 L}=3, \quad$ and $c_{0 C}=-4 \kappa\left(J_{P} / F_{p}^{2}\right)$. This is a cubic equation in the variable $V^{-1 / 2}$; the terms using coefficients $c_{1 L}$ and $c_{3 L}$ dominate in the low $-\zeta$ situation, the terms using coefficients $c_{0 C}$ and $c_{3 C}$ dominate in the high- $\zeta$ (Child's law) situation.

Further substitutions $a_{1}=c_{1 L} /\left(c_{3 C}+c_{3 L}\right) \quad$ and $a_{0}=c_{0 C} /\left(c_{3 C}+c_{3 L}\right)$ yield

$$
G(V) \equiv V^{-3 / 2}+a_{1} V^{-1 / 2}+a_{0}=0 .
$$

This is a standard mathematical form for which an analytical solution exists (see Appendix 1).

A value is now needed for $\beta_{L}$, and the relevant emission equation $J_{P}\left(F_{P}\right)$ must be introduced. Barbour et al. ${ }^{9}$ took $\beta_{L}$ to be the value given by Eq. (2) in the real experimental situation. This $\beta_{L}$ can in principle be calculated from the system geometry, but for CFE, it is better obtained empirically from the slope of the linear middle region of a FN plot of type $\left[\ln \left\{i / V^{2}\right\}\right.$ versus $\left.1 / V\right]$.

A convenient procedure ${ }^{9}$ is then to choose a suitable value of $F_{P}$, calculate corresponding values of $J_{P}, a_{0}$, and $a_{1}$, and solve Eq. (28) to obtain the corresponding value of $V$. In the past, analytical solutions have been thought awkward, so computational methods have been used. If the dependence of notional emission area $A$ on voltage is known, or if $A$ is taken to have a known constant value, then the emission current $i$ can be obtained from $i=A J_{P}$. This yields a consistent set of values $\left(F_{P}, J_{P}, V, i\right)$. From many such sets, data for a $J_{P}(V)$ or $i(V)$ characteristic is obtained. This approach was used by Barbour et al., ${ }^{9}$ specifically for CFE. However, the equations here apply to any charged-particle surface emission process, once the relevant emission equation $J_{P}\left(F_{P}\right)$ is provided.

\section{ILLUSTRATIVE APPLICATIONS}

To illustrate formula use, we examine some simple applications. As earlier, $J_{P}$ denotes the actual emission current

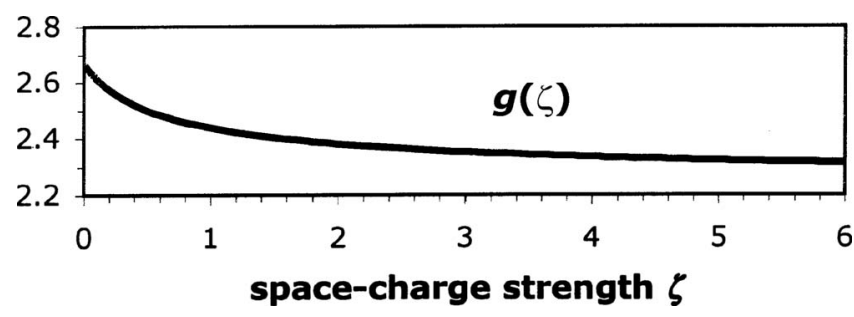

FIG. 2. To show how the factor $g(\zeta)$ varies with $\zeta$.

density, $J_{L}$ the corresponding Laplace current density, and $J_{C}$ the corresponding Child's law current density. The predictions of $J_{L}$ and $J_{C}$ are made for the same values of $\beta_{L}, V$, and $F_{L}$ as applied to the calculation of $J_{P}$ (i.e., they are made for the same system geometry and applied voltage).

\section{A. Field reduction at the LMIS apex}

For the gallium LMIS, the operating point on the $i(V)$ characteristic is controlled by FEVSC effects. ${ }^{20}$ Near emission onset, the LMIS apex radius $r_{a}$ is typically about 1.5 $\mathrm{nm}$, and the emission current typically about $2 \mu \mathrm{A} .{ }^{36}$ Taking emission to come from an area $\pi r_{a}^{2}\left(\approx 7.1 \mathrm{~nm}^{2}\right)$, we estimate current density as $J_{P} \approx 2.8 \times 10^{11} \mathrm{~A} / \mathrm{m}^{2}$.

Planar FEVSC theory does not strictly apply to such a small sharply curved object, but can be used to estimate $\zeta$ and $\theta$. For $\mathrm{Ga}^{+}$ions, $\kappa \cong 6.789 \times 10^{7} \mathrm{~A} \mathrm{~V}^{3 / 2}$. The LMIS emission mechanism is field evaporation, which for Ga occurs at $F_{P} \approx 15 \mathrm{~V} / \mathrm{nm}^{36}$ A typical extracting voltage is $V=3000 \mathrm{~V}$. From Eq. (11), these numbers yield $\zeta \approx 4.7$, $S \approx 1370$, and $\theta \approx 0.29$. This strongly supports the idea that substantial field reduction occurs at a LMIS apex.

\section{B. Field-stress reduction at the LMIS apex}

A surface field $F$ causes an outward electrostatic ("Maxwell") stress $M$ on the surface, given by $M=\frac{1}{2} \varepsilon_{0} F^{2}$. For a given system geometry and applied voltage, the Maxwell stress in the presence of space charge $\left(M_{P}\right)$ is less than it would have been $\left(M_{L}\right)$ in the absence of space charge, by an amount $\Delta_{M}$ given by

$$
\Delta_{M}=M_{L}-M_{P}=M_{P}\left(\theta^{-2}-1\right) .
$$

If a parameter $g(\zeta)$ is defined by

$$
g(\zeta) \equiv\left(\theta^{-2}-1\right) / \zeta
$$

so $\Delta_{M}=g(\zeta) \zeta M_{P}$, then Eqs. (16), (20), and (30) show that, in the limiting cases where $\zeta$ has low and high values, we obtain $g=8 / 3$ and $9 / 4$, respectively.

The quantities $\Delta_{M}$ and $g(\zeta)$ are important when deriving Mair's equation $^{20,36}$ for the LMIS $i(V)$ characteristic. Since we now have a relationship $\theta(\zeta)$ valid for the whole range of $\zeta$, Eq. (28) can be used to plot $g(\zeta)$ up to $\zeta$-values beyond those likely to be found in practice. The result is Fig. 2. This confirms that the variation is smooth and small, with no hidden difficulties.

The results in Secs. V A and V B have been used in a recent review of LMIS theory. ${ }^{36}$ 


\section{Effect of FEVSC on Fowler-Nordheim plot shape}

We now illustrate electron emission effects, starting with the shape of FN plots. ${ }^{5,47}$ FEVSC effects on CFE have often been discussed (see earlier citations), but often, following Barbour et al., predictions are presented as semilogarithmic plots, of type $\left[\ln \{J\}\right.$ versus $\left.1 / F_{L}\right]$ or equivalent. The predicted behavior of FN plots in strong space-charge conditions has not been fully described.

For metals, CFE is described in principle by the socalled physical (or "physically complete") FN-type equation, ${ }^{58}$ written here in the form used in Eq. (18) of Ref. 58 , namely,

$$
J_{P}=J_{\mathrm{FN}}=\lambda_{Z} a \phi^{-1} F_{p}^{2} P_{F} \exp \left(-\nu_{F} b \phi^{3 / 2} / F_{P}\right) .
$$

Here $\phi$ is the local work function of the emitting surface, $a\left(\cong 1.541434 \times 10^{-6} \mathrm{~A} \mathrm{eV} \mathrm{V}^{-2}\right) \quad$ and $\quad b(\cong 6.830890$ $\mathrm{eV}^{-3 / 2} \mathrm{~V} \mathrm{~nm}^{-1}$ ) are the first and second FN constants as usually defined, ${ }^{47} \nu_{F}$ is a physical correction factor associated with the barrier-shape model used, ${ }^{58} P_{F}$ is a tunnelling prefactor, ${ }^{59}$ and $\lambda_{Z}$ is a physical correction factor associated with electron supply ${ }^{58}$ to the barrier. To make calculations practicable, we used the so-called standard FN-type equation ${ }^{46,47}$ in which the correction factors in Eq. (31) have been approximated in the following way: $P_{F} \rightarrow 1, \nu_{F} \rightarrow v_{F}$, and $\lambda_{Z} \rightarrow \tau_{F}^{-2}$, where $v_{F}$ and $\tau_{F}$ are the relevant values of mathematical functions (the "Schottky-Nordheim barrier functions") well known ${ }^{46,47}$ in CFE theory. These replacements yield the equation

$$
J_{P} \approx \tau_{F}^{-2} a \phi^{-1} F_{p}^{2} \exp \left[-v_{F} b \phi^{3 / 2} / F_{P}\right] .
$$

The analytical solution of Eq. (28) was then implemented on a spreadsheet (see Appendix 1), using Eq. (32). It is thought that analytical solutions for Eq. (28) have not previously been used in CFE literature; Appendix 1 notes some mathematical details.

Figure 3 shows the resulting FN plot, for $\phi=4.5 \mathrm{eV}$, and the Barbour et al. ${ }^{9}$ value of $\beta_{L}=4 \times 10^{5} \mathrm{~m}^{-1}$. Curve $L$ is from the emission equation [Eq. (32)] in the absence of space charge, curve $C$ is from Child's law [Eq. (21)], and curve $P$ (marked with dots) is from Eq. (28). Following Barbour et al. ${ }^{9}$ these plots are made against $1 / F_{L}\left[\equiv 1 / \beta_{L} V\right]$, rather than against $1 / V$, because (for a given $\phi$-value) curve $L$ is then the same for all $\beta_{L}$ values. Clearly, as $F_{L}$ increases $\left(1 / F_{L}\right.$ decreases), curve $P$ initially follows curve $L$, then separates from it and "turns over completely" to join curve $C$.

Curve $P$ is evaluated only up to the field value $F_{P}=F_{b}$ where the top of tunneling barrier goes below the Fermi level, and is terminated at the value of $F_{L}$ corresponding to $F_{P}=F_{b}$. Above $F_{P}=F_{b}$ the emission regime becomes fieldinduced ballistic emission; the theory of this emission regime is not well developed, although there is a recent exploration. $^{60}$

In fact, Eq. (32) ceases to be physically valid at a value of $F_{P}$ slightly lower than $F_{b}$; an alternative relationship takes over, and extends beyond $F_{b}$, producing a "high-field intermediate emission regime." Causes of breakdown of Eq. (32), as $F_{P}$ approaches $F_{b}$, are failure of the image potential energy to correctly represent correlation-and-exchange

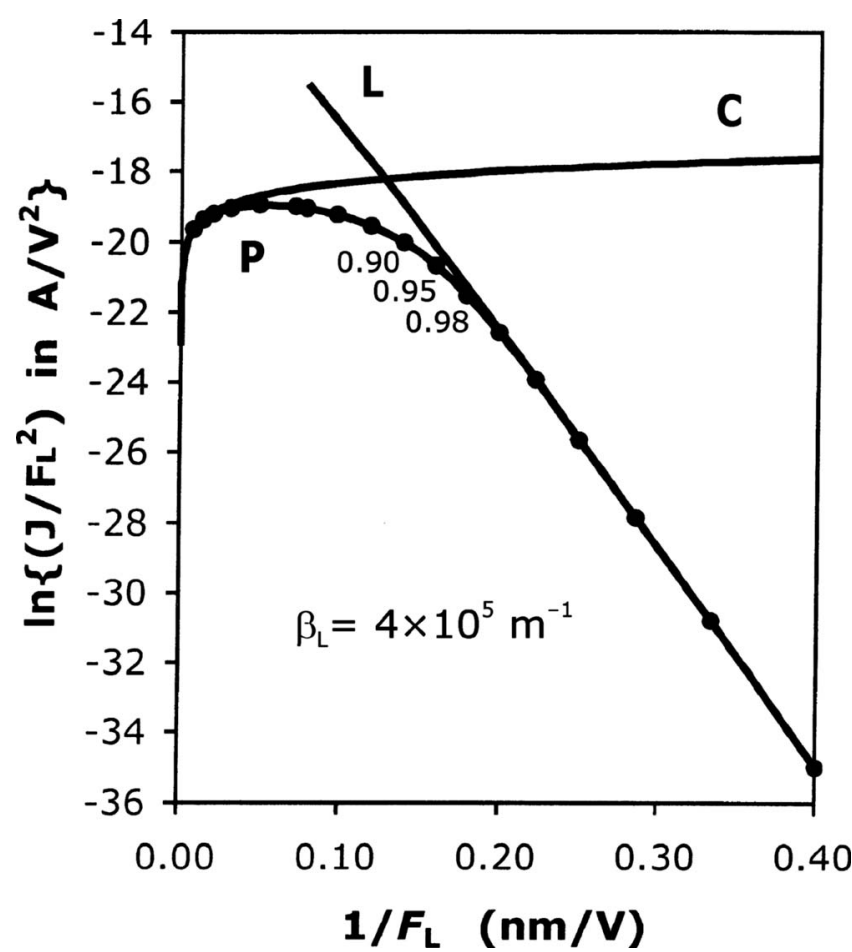

FIG. 3. Plots, in FN coordinates, showing how the Laplace $(L)$, Poisson $(P)$, and Child's law $(C)$ current densities $J_{L}, J_{P}$, and $J_{C}$ vary with the Laplace field $F_{L}$, for the parameter values $\phi=4.5 \mathrm{eV}$ and $\beta_{L}=4 \times 10^{5} \mathrm{~m}^{-1}$. This $\beta_{L}$ value corresponds to a tungsten emitter of moderate to large apex radius, in a conventional field electron microscope configuration. Curve $P$ is marked with dots and is terminated at the point where the Poisson field $F_{P}$ becomes equal to the value $F_{b}$ at which the top of the tunneling barrier goes below the emitter Fermi level. The numbers 0.90, 0.95, and 0.98 are $\theta$-values corresponding to different possible criteria for the onset of FEVSC effects, and label points on the Poisson curve where $\theta$ approximately has these values.

effects, ${ }^{48,49,61,62}$ field-induced changes in surface wave functions that lead to a change in work function, ${ }^{62}$ breakdown, when the barrier height is small, of the simple JefferiesWentzel-Kramers-Brillouin formula used in the derivation of Eq. (32), ${ }^{59,63}$ and/or (close to $F_{P}=F_{b}$ ) increasing incidence of thermally activated electron escape over the top of the barrier. As the references indicate, some of these effects have been investigated individually; but no integrated emission theory exists for this high-field intermediate regime.

In practice, these theoretical weaknesses in emission theory cause no qualitative difficulty for curve $P$ in Fig. 3, because near $F_{P}=F_{b}$ a corrected curve $P$ would (almost certainly) closely resemble curve $C$. A theoretically correct curve $P$ would certainly turn over completely as $V$ and $F_{L}$ increase, but its shape in the turn-over region would be slightly different.

Curve $L$ is terminated at $F_{L}=F_{b}$. For visual convenience, curve $C$ is arbitrarily terminated at $1 / F_{L}=10^{-5} \mathrm{~nm} / \mathrm{V}$. Obviously, this corresponds to $F_{L}$ values enormously above those accessible experimentally. Note that both the Poisson and Laplace curves do in principle extend to higher field values than $F_{b}$; they are terminated in the figures because, as yet, we have no satisfactory mathematical emission theory for this field range.

Some past FEVSC analyses have used, instead of Eq. (32), the so-called "elementary FN-type equation." This is 


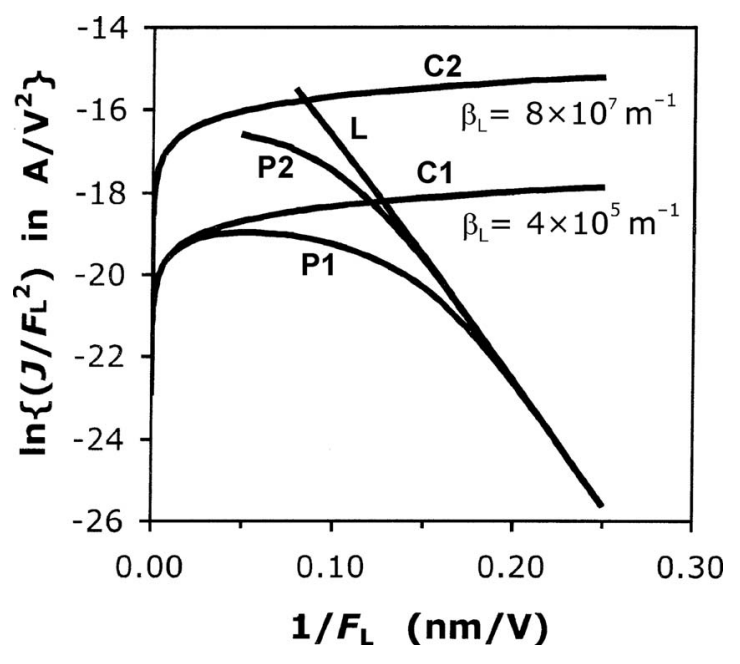

FIG. 4. Enlarged version of the turn-over region in Fig. 3, for $\beta_{L}$ values (1) $4 \times 10^{5} \mathrm{~m}^{-1}$ and (2) $8 \times 10^{7} \mathrm{~m}^{-1}$. The latter value corresponds to a sharp emitter, as might be used in a Spindt array. The Laplace and Poisson curves are terminated when the top of the relevant tunneling barrier goes below the emitter Fermi level. For the high- $\beta_{L}$ Poisson curve this occurs before turn over. Theoretically, the Poisson curves continue into the ballistic emission regime and eventually merge with the relevant Child's law curve, but this part of the Poisson curve may not normally be experimentally accessible. Figure 4 suggests that, for sharp emitters, FEVSC effects become significant at higher current densities than for blunter emitters, if other things are equal.

obtained from Eq. (28) by the replacements $P_{F} \rightarrow 1, \nu_{F} \rightarrow 1$, and $\lambda_{Z} \rightarrow 1$, and is used to describe CFE in undergraduate textbooks. Use of this elementary equation will not yield a correct physical analysis at high fields, because it does not allow the top of the barrier to go below the Fermi level, so no field-induced ballistic emission regime is predicted.

The complete turn over of curve $P$, as $V$ and $F_{L}$ increase, initially came as a surprise to the author. In fact, the effect could have been predicted from Fig. 9 in Ref. 27, and a similar trend is evident in Fig. 2 of Ref. 25, although the effect is more obvious in the present calculations. Complete turn over at sufficiently high voltage can also be predicted by a simple theoretical argument. In the Child's law regime, Eq. (21) shows that $J_{C} \sim V^{3 / 2}$, hence $J_{C} \sim F_{L}^{3 / 2}$. It follows that $\left(J_{C} / F_{L}^{2}\right) \sim\left(1 / F_{L}\right)^{1 / 2}$, so one expects curve $C$ to have the shape shown, and curve $P$ to approximate this shape at low values of $1 / F_{L}$.

In FN coordinates of type $\left[\ln \left\{J / F_{L}^{2}\right\}\right.$ versus $\left.1 / F_{L}\right]$, Child's law has the form

$$
\ln \left\{J_{C} / F_{L}^{2}\right\}=\ln \left\{\left(\frac{4}{9}\right) \kappa^{-1} \beta_{L}^{1 / 2}\right\}+\left(\frac{1}{2}\right) \ln \left\{1 / F_{L}\right\} .
$$

Curves for different $\beta_{L^{-}}$-values are parallel vertically, so the limiting form of curve $P$ at low values of $1 / F_{L}$ depends on $\beta_{L}$. For example, individual emitters in a Spindt array have $\beta_{L^{-}}$-values typically around $10^{7}-10^{8} \mathrm{~m}^{-1}$ (compare the Barbour et al. ${ }^{9}$ value of $4 \times 10^{5} \mathrm{~m}^{-1}$ ). So, for a Spindt emitter the maximum will tend to occur at values of $\ln \left\{J_{P} / F_{L}^{2}\right\}$ and $J_{P}$ higher than those for a moderate-radius tungsten emitter. Figure 4 illustrates this effect, for the relatively high value $\beta_{L}=8 \times 10^{7} \mathrm{~m}^{-1}$.

Thus, for $\phi=4.5 \mathrm{eV}$ and $\beta_{L}=4 \times 10^{5} \mathrm{~m}^{-1}$, the maximum in curve $P$ occurs at values $F_{P} \approx 9.2 \mathrm{~V} / \mathrm{nm}$, $J_{P} \approx 3.0 \times 10^{12} \mathrm{~A} / \mathrm{m}^{2}$. However, for $\phi=4.5 \mathrm{eV}$ and $\beta_{L}=8 \times 10^{7} \mathrm{~m}^{-1}$, the maximum is predicted to occur at field
$F_{P}$ greater than $F_{b}[>14.0 \mathrm{~V} / \mathrm{nm}]$; if standard theory were quantitatively valid at this point (which it is not), then we would predict that $J_{P}>5.5 \times 10^{13} \mathrm{~A} / \mathrm{m}^{2}$.

As noted earlier, the emission current is $i=A J_{P}$, where $A$ is the notional emission area. Obviously, with raw experimental data, one plots $\ln \left\{i / V^{2}\right\}$ against $1 / V$. If $A$ is taken as constant, such plots are predicted to have the same general shape as curve $P$. However, as far as the author is aware, experimental FN plots exhibiting complete turn over have never been reported. Probably, the main reason has usually been that the maximum in curve $P$ occurs at voltages higher than those that could be safely applied in the experiments in question. (With real field emitters, the current densities may often not be able to get near the Child's law situation, even with pulsed operation, because heating would be too great and destroy the emitter.) This was certainly the case with the Barbour et al. ${ }^{9}$ experiments and the similar experiments by Dyke and Trolan, ${ }^{64}$ where the $\beta_{L}$-values are known. Other possible reasons for nonobservation of turn over are that data have not been plotted in the correct form to show the effect, or that the emission area $A$ depends on voltage, with a power law greater than 0.5 (voltage dependence in $A$ was suggested long $\mathrm{ago}^{65}$ ).

Given that new sharper types of emitter have been introduced since serious experiments were last performed on FEVSC theory, it might be interesting to measure pulsed highvoltage $i(V)$ characteristics for emitters able to withstand high current densities for short times (if experimental problems can be overcome ${ }^{66,67}$ ). A difficulty is that a small-radius emitter is better able to dissipate heating effects, but exhibits FEVSC effects only at higher $J_{P}$-values: At present, the trade-off between these effects is not clear. A second difficulty is that emitter heating will by itself change the emission current density, and this change will need to be taken into account if there is a significant rise in apex temperature.

\section{Criterion for onset of space-charge effects}

The current density at which FEVSC effects cause observable deviation from FN plots or Barbour et al. ${ }^{9}$ type plots is of interest. A possible criterion is $\theta=0.90$. In Fig. 3, this occurs when the horizontal separation of curves $L$ and $P$ is roughly $10 \%$ of the $X$-axis value for curve $L$. (Points corresponding approximately to $\theta=0.90,0.95$, and 0.98 are marked.)

Equation (15) allows an approximate treatment. For $\theta$ $=0.90$ we obtain $\zeta=\kappa V^{1 / 2} J_{P} / F_{P}^{2}=0.08965$. With $\kappa=1.9043$ $\times 10^{5} \mathrm{~A} \mathrm{~V}^{3 / 2}$, and for $V=20 \mathrm{kV}$ (typical of the Barbour et al. ${ }^{9}$ experiments), we obtain $J_{P} / F_{P}^{2} \sim 3.3 \times 10^{-9} \mathrm{~A} \mathrm{~V}^{-2}$. The formula $F_{P}=\theta \beta_{L} V$ yields $F_{P} \sim 7.2 \mathrm{~V} / \mathrm{nm}$ and then $J_{P} \sim 1.7$ $\times 10^{11} \mathrm{~A} / \mathrm{m}^{2}$.

For comparison, the exact spreadsheet-based planargeometry calculation, with $\phi=4.5 \mathrm{eV}$ and $\beta=4 \times 10^{5} \mathrm{~m}^{-1}$, yields $F_{P} \approx 6.7 \mathrm{~V} / \mathrm{nm}, J_{P} \approx 1.6 \times 10^{11} \mathrm{~A} / \mathrm{m}^{2}, \ln \left\{J_{P} / F_{L}^{2}\right\} \approx$ -19.7 , and $V \approx 18700 \mathrm{~V}$. It also finds the vertical difference between curves $L$ and $P$ at this point as about 1.1 Np.

For a small-radius emitter such as a Spindt emitter, the illustrative values $\phi=4.5 \mathrm{eV}, \beta_{L}=8 \times 10^{7} \mathrm{~m}^{-1}$, and $V$ $=100 \mathrm{~V}$ lead to the approximate results: $F_{P} \sim 7.2 \mathrm{~V} / \mathrm{nm}$ and 
$J_{P} \sim 2.4 \times 10^{12} \mathrm{~A} / \mathrm{m}^{2}$. The exact planar geometry calculation yields $F_{P} \approx 9.4 \mathrm{~V} / \mathrm{nm}, \quad J_{P} \approx 3.7 \times 10^{12} \mathrm{~A} / \mathrm{m}^{2}$, $\ln \left\{J_{P} / F_{L}^{2}\right\} \approx-17.2$, and $V \approx 131.2 \mathrm{~V}$, with the vertical difference between curves $A$ and $B$ equal to $0.8 \mathrm{~Np}$.

Figure 4 compares these cases, and suggests that FEVSC effects set in at significantly higher current densities for emitters with high $\beta_{L}$ values. Physically, this can be deduced from the definition of FEVSC strength, $\zeta=\kappa V^{1 / 2} J_{P} / F_{P}^{2}$ $=\kappa\left(F_{L}^{1 / 2} / F_{P}^{2}\right)\left(\beta_{L}^{-1 / 2} J_{P}\right)$. If we disregard changes in field in comparison with changes in $J_{P}$, then a given value of $\zeta$ is achieved at a given value of $\left(\beta_{L}^{-1 / 2} J_{P}\right)$. This implies that (in a first approximation) the $J_{P}$ value for onset of FEVSC effects is proportional to $\beta_{L}^{1 / 2}$. Sharp emitters have high $\beta_{L}$ values, so (to the extent that $1 \mathrm{D}$ planar theory applies) one expects FEVSC effects to become significant for sharp emitters at higher $J_{P}$ values than for blunt emitters.

Numerically, it seems clear that estimates made using the spreadsheet program are more reliable than those made using simple arguments based on Eq. (15). This is probably because the latter approach involves estimating the onset voltage for FEVSC effects.

\section{E. Criterion for approach to the Child's law regime}

A simple criterion for approach to the full Child's law regime puts $J_{P} / J_{C}$ equal to some set fraction. For illustration, we use $J_{P} / J_{C}=0.80$ here. This corresponds to a separation between curves $C$ and $P$ of about 0.22 Np. From Eqs. (24) and (25) we find that this occurs for $\theta=0.3963$ and $\zeta$ $=2.264$.

For $\phi=4.5 \mathrm{eV}$ and $\beta_{L}=5 \times 10^{4} \mathrm{~m}^{-1}$, spreadsheet calculations show that this criterion corresponds to $F_{P}$ $=9.70 \mathrm{~V} / \mathrm{nm}, \quad F_{L}=24.5 \mathrm{~V} / \mathrm{nm}, \quad V=61 \mathrm{kV}, \quad J_{P}=4.5$ $\times 10^{12} \mathrm{~A} / \mathrm{m}^{2}$, and $\ln \left\{J_{P} / F_{L}^{2}\right\}=-18.7$.

For $\phi=4.5 \mathrm{eV}$ and $\beta_{L}=8 \times 10^{7} \mathrm{~m}^{-1}$, spreadsheet calculations show that this criterion corresponds to a surface field $F_{P}$ significantly greater than $F_{b}$ (i.e., $F_{P}$ significantly greater than $14.0 \mathrm{~V} / \mathrm{nm}, F_{L}$ significantly greater than $35 \mathrm{~V} / \mathrm{nm}$, and $J_{P}$ significantly greater than $\left.5.5 \times 10^{13} \mathrm{~A} / \mathrm{m}^{2}\right)$. So, according to planar FEVSC theory, an emitter with $\phi=4.5 \mathrm{eV}$ and $\beta_{L}=8 \times 10^{7} \mathrm{~m}^{-1}$ that is emitting in the CFE emission regime can never be really close to the Child's law space-charge regime. At the field $F_{b}$ we have $\theta \approx 0.55$ and $J_{P} / J_{C} \approx 0.64$.

Obviously, the numerical results obtained here and in Sec. V E depend on the precise criteria chosen. As yet, there is no consensus on what criteria should be used. Also, of course, the theory here is a theory of what happens if the emitter neither heats significantly nor self-destructs. Heating issues (especially for sharp emitters) are considered in Fursey's ${ }^{66}$ book, and also motivate Jensen's ${ }^{63}$ work on the development of general equations covering thermal, field, and photoemission.

\section{A MODIFIED PLANAR-GEOMETRY MODEL}

In the plane-parallel geometry used above, the emitter surface sees the field due to a FEVSC "cone" of solid angle $2 \pi$. With a real field emitter, the local current density falls off with distance from the surface, and the surface sees the field due to a FEVSC cone of solid angle much less than $2 \pi$.
So, for given surface emission current density, the FEVSCinduced reduction in surface field is expected to be less for the nonplanar geometry.

In formulas above, the strength of FEVSC effects could be changed by replacing $\zeta$ by

$$
\zeta^{c}=\omega \zeta=\omega \kappa V^{1 / 2} J F_{P}^{-2},
$$

where $\omega$ is a "strength correction factor." Thus, for example, for the small-space-charge regime we would obtain

$$
\theta=1-(4 / 3) \omega \zeta \text {. }
$$

If the correct $\theta(\zeta)$ dependence were known at the emitter apex for some specific system geometry, then for any specific $\theta$-value used as a criterion, one could use $\omega$ as a scaling parameter to make Eq. (35) predict the correct answer. Appropriate values of $\omega$ are not currently known, and it is not known how good this "scaling" would be for other $\zeta$-values. It would seem useful to explore these issues. The means to do this seems available since FEVSC effects are already embedded in some academic and commercial programs, ${ }^{37-40}$ based on Monte Carlo methods, that are used to analyze electron and ion optical effects in the design of focusedbeam systems.

More generally, Mair's equation, ${ }^{20,36}$ for the LMIS $i(V)$ characteristic, is quantitatively successful in predicting $d i / d V$, even though its derivation involves applying a planargeometry FEVSC formula to an emitting apex of radius about $1.5 \mathrm{~nm}$. Thus, there seems some hope that useful approximate formulas for nonplanar geometries might be devised. I urge that relevant Monte Carlo simulations involving realistic emitter geometries be carried out.

\section{RELATIVISTIC EFFECTS}

Electron mass increases at relativistic speeds. For given applied voltage, electrons take slightly longer to leave the vacuum space than nonrelativistic FEVSC theory would predict; so the FEVSC-induced reduction in emitter field is slightly greater. For CFE from metals, the theory of this effect is well explained in a paper by Wheeler. ${ }^{16}$

The energy-equivalent $E_{e}$ of the electron rest mass $\left(E_{e}\right.$ $=m_{e} c_{0}^{2}$, where $c_{0}$ is the velocity of light) is about $250 \mathrm{keV}$. At an energy $E_{c} / 10$, i.e., about $25 \mathrm{keV}$, the increase in mass is about $10 \%$. From Eqs. (5) and (11), the equivalent increases in $\kappa$ and $\zeta$ are about 5\%. However, the actual effect on calculated values of $\theta$ and $F_{P}$ will be less than this, because relativistic mass increase will be significant only when the electron has reached a sufficiently large speed. For working purposes, we can take $25 \mathrm{kV}$ as the voltage value above which relativistic corrections begin to need consideration.

For real electron emitters, few applications will use extraction voltages as high as $25 \mathrm{kV}$, so relativistic corrections will often not be relevant. However, in accelerator and other high-voltage contexts, depending on the precise arrangements of electrodes and voltages, relativistic effects may need thinking about.

On the other hand, theoretical predictions of FEVSC effects (including those here) certainly may extend formally into field and voltage ranges where relativistic corrections 
ought in principle to be included, if quantitative selfconsistency is needed. For example, the values of the Laplace field $F_{L}$ corresponding to a voltage of $25 \mathrm{kV}$ are: 10 $\mathrm{V} / \mathrm{nm}$ for $\beta_{L}=4 \times 10^{5} \mathrm{~m}^{-1}$ and $2000 \mathrm{~V} / \mathrm{nm}$ for $\beta_{L}=8$ $\times 10^{7} \mathrm{~m}^{-1}$. Since the real emitter surface field $F_{P}$ must be less than $F_{L}$, it follows that, for the emitters of moderate apex radius to which the value $\beta_{L}=4 \times 10^{5} \mathrm{~m}^{-1}$ applies, the formal predictions shown in Fig. 3 do reach into a field range $\left(1 / F_{L}<0.1 \mathrm{~nm} / \mathrm{V}\right)$ where relativistic corrections are relevant in principle.

However, relativistic corrections do not significantly affect the qualititative arguments about curve turn over presented here, because they tend to reduce the emission current below the value predicted by Child's law, and hence tend to enhance the turn-over effect seen in a FN plot.

Relativistic effects are thought to be unimportant for field ion emission sources because ion masses are much higher than the electron mass.

\section{SUMMARY}

This paper has aimed to put onto a better mathematical basis the classical 1D theory of field-emitted vacuum space charge in planar geometry. Three dimensionless parameters $(\zeta, \theta$, and $j)$, and the two simple dimensionless relationships (13) and (23) are the core of this revised FEVSC mathematics. From these, we can derive most existing basic FEVSC formulas and some additional ones. A key feature has been the introduction of the concept of space-charge strength $\zeta$.

Illustrative applications have been: (a) formulas (14a) and (14b) enable the magnitude of field reduction effects to be estimated easily, (b) formula (30) fills in a missing part of liquid metal ion source theory, (c) the procedure described in Appendix 1 enables $J$ - $V$ characteristics to be evaluated analytically, (d) Fig. 3 shows that related FN plots may be shaped differently from common expectation, and (e) Fig. 4 suggests that high- $\beta_{L}$ emitters will be less affected by FEVSC.

These results should be seen primarily as contributing to better basic qualitative understanding of FEVSC effects. However, they also have quantitative value as limits. This is because we expect that, for a given space-charge strength, the planar-geometry situation is the "worst case," in the sense that FEVSC effects will be greater in this geometry than in configurations involving curved emitters.

If possible, it would be desirable to develop simple FEVSC approximations and formulas that apply adequately to more realistic emitter geometries. These should supplement, rather than replace, detailed numerical investigations. It is suggested that the sophisticated Monte Carlo-type programs used in electron and ion optical designs could be customized to investigate FEVSC effects in realistic system geometries, and it is urged that this possibility be explored.

\section{APPENDIX: SOLUTION OF CUBIC EQUATION FOR $J_{P}(V)$.}

General analytical procedures for solving cubic equations are well known and described in textbooks and on the web. ${ }^{68}$ A problem with Eq. (28) is to identify unambiguously, at any particular voltage, which of the mathematical roots corresponds to the physical solution. This problem is usually avoided by solving the equation numerically and tracking the lowest positive root as $V$ increases. ${ }^{9}$ This appendix indicates how to deal with it when using analytical solutions. Earlier FEVSC literature has not discussed this.

Equation (28) is a standard form for a cubic equation in the variable $z \equiv V^{-1 / 2}$, and has analytical solutions as follows: ${ }^{68}$

$$
\begin{aligned}
& R \equiv-a_{0} / 2, \quad D \equiv a_{1}^{3} / 27+a_{0}^{2} / 4, \\
& S \equiv(R+\sqrt{ } D)^{1 / 3}, \quad T \equiv(R-\sqrt{ } D)^{1 / 3} .
\end{aligned}
$$

In the case where $D<0$, and $\sqrt{ } D$ is imaginary, we may use standard procedures to evaluate specific roots for $S$ and $T$ in the form

$$
S \equiv X+j Y, \quad T \equiv X-j Y,
$$

where $j \equiv \sqrt{ }-1, X$, and $Y$ are positive real numbers and $S$ lies in the first quadrant of the Argand diagram. In these circumstances, the three roots of Eq. (28) are all real and are given by

$$
\begin{aligned}
& V_{1}^{-1 / 2}=2 X, \\
& V_{2}^{-1 / 2}=-X-Y \sqrt{ } 3, \\
& V_{3}^{-1 / 2}=-X+Y \sqrt{ } 3 .
\end{aligned}
$$

The root $V_{2}^{-1 / 2}$ is negative and of no physical interest.

In the case where $D>0$, and $\sqrt{ } D$ is real, there are one real root and two complex roots, with the real root given by

$$
V_{4}^{-1 / 2}=S+T=(R+\sqrt{ } D)^{1 / 3}+(R-\sqrt{ } D)^{1 / 3},
$$

where $S$ is a positive real number and $T$ a negative real number.

It has been straightforward to program the results for $V_{1}$, $V_{3}$, and $V_{4}$, and the corresponding values of $\theta$, onto a spreadsheet developed from one described earlier, ${ }^{69}$ which evaluates $J_{P}\left(F_{P}, \phi\right)$ for the standard $\mathrm{FN}$-type equation. Inspection of trends, as the values of $F_{P}$ (and hence $V$ ) increase from zero, shows that there are two mathematical branch points and an emission regime change point (where the top of the tunneling barrier goes below the Fermi level). At low $V$-values the physical solution is $V_{1}$. At the lower branch point (which corresponds to the one already identified in Eq. (15), at which $S=0$ ), the physical solution changes from $V_{1}$ to $V_{3}$. At the upper branch point, which corresponds to the point where $D$ changes from negatively infinite to positively infinite, the physical solution changes from $V_{3}$ to $V_{4}$.

For an emitter with $\phi=4.5 \mathrm{eV}$ and $\beta_{L}=4 \times 10^{5} \mathrm{~m}^{-1}$, the branch points and the change point occur at fields of 8.105 , 8.491 , and $14.063 \mathrm{~V} / \mathrm{nm}$, respectively. The same pattern applies for other values of $\phi$ and $\beta_{L}$, but with different field values.

One needs to select the physically appropriate branch for any particular $F_{P}$-value, without inspecting trends. Identifying the upper branch point is easy, as $V_{4}^{-1 / 2}$ changes from complex to real at this point. The lower branch point is less straightforward. However, it is found that $D\left(F_{P}\right)$ goes 
through a maximum value of $D=0$ at this branch point. So a convenient approach is to test the signs of $D$ and $d D / d F_{P}$ : branch $V_{1}$ is the physical solution if $D<0$ and $d D / d F_{P}>0$, branch $V_{3}$ is the physical solution if $D<0$ and $d D / d F_{P}<0$, and branch $V_{4}$ is the physical solution if $D>0$. The value of $d D / d F_{P}$ is found numerically.

These remarks apply specifically to the solution of Eq. (28) in the context of FEVSC, and are not intended to apply generally to cubic equations.

${ }^{1}$ C. D. Child, Phys. Rev. 34, 492 (1911).

${ }^{2}$ I. Langmuir, Phys. Rev. 2, 450 (1913).

${ }^{3}$ I. Langmuir, Phys. Rev. 21, 419 (1923).

${ }^{4}$ G. Jaffé, Ann. Phys. (Leipzig) 63, 145 (1920).

${ }^{5}$ T. E. Stern, B. S. Gossling, and R. H. Fowler, Proc. R. Soc. London, Ser. A 124, 699 (1929).

${ }^{6}$ R. H. Fowler and L. W. Nordheim, Proc. R. Soc. London, Ser. A 119, 173 (1928).

${ }^{7}$ R. Cockburn, Proc. Phys. Soc. London 47, 810 (1935).

${ }^{8}$ H. F. Ivey, Phys. Rev. 76, 554 (1949).

${ }^{9}$ J. P. Barbour, W. W. Dolan, J. K. Trolan, E. E. Martin, and W. P. Dyke, Phys. Rev. 92, 45 (1953).

${ }^{10}$ L. Page and N. I. Adams, Phys. Rev. 76, 381 (1949).

${ }^{11}$ H. F. Ivey, Adv. Electron. Electron Phys. 6, 137 (1954).

${ }^{12}$ I. Itzkan, J. Appl. Phys. 31, 652 (1960).

${ }^{13}$ P. A. Chatterton, Proc. Phys. Soc. 88, 231 (1966).

${ }^{14}$ R. Nowicki, Surf. Sci. 8, 357 (1967).

${ }^{15}$ C. B. Wheeler, J. Phys. A 6, 1439 (1973).

${ }^{16}$ C. B. Wheeler, J. Phys. D 7, 267 (1974).

${ }^{17}$ B. W. Weinstein, Ph.D. thesis, University of Illinois at UrbanaChampaign, 1975.

${ }^{18}$ N. M. Miskovsky and P. H. Cutler, Appl. Phys. 28, 73 (1981).

${ }^{19}$ G. L. R. Mair, J. Phys. D 15, 2623 (1982).

${ }^{20}$ G. L. R. Mair, J. Phys. D 17, 2323 (1984).

${ }^{21}$ J. Fernández de la Mora, J. Fluid Mech. 243, 561 (1992).

${ }^{22}$ W. A. Anderson, J. Vac. Sci. Technol. B 11, 383 (1993).

${ }^{23}$ Z. Cui and L. Tong, IEEE Trans. Electron Devices 40, 448 (1993).

${ }^{24}$ I. Brodie and P. R. Schwoebel, Proc. IEEE 82, 1006 (1994).

${ }^{25}$ G. N. A. van Veen, J. Vac. Sci. Technol. B 12, 655 (1994).

${ }^{26}$ Y. Y. Lau, Y. Liu, and R. K. Parker, Phys. Plasmas 1, 2082 (1994).

${ }^{27}$ K. L. Jensen, P. Mukhopadhyay-Phillips, E. G. Zaidman, K. Nguyen, M. A. Kodis, L. Malsawma, and C. Hor, Appl. Surf. Sci. 111, 205 (1997).

${ }^{28}$ K. L. Jensen, M. A. Kodis, R. A. Murphy, and E. G. Zaidman, J. Appl. Phys. 82, 845 (1997)

${ }^{29}$ A. K. Batrakov, I. V. Pegel, and D. I. Proskurovskii, Tech. Phys. Lett. 26, 454 (1998).

${ }^{30}$ P. V. Akimov, H. Schamel, H. Kolinsky, A. Ya. Ender, and V. I. Kuznetsov, Phys. Plasmas 8, 3788 (2001).

${ }^{31}$ R. A. Murphy and M. A. Kodis, in Vacuum Microelectronics, edited by W. Zhu (Wiley, New York, 2001).

${ }^{32}$ V. G. Pavlov, Tech. Phys. 49, 1610 (2004).

${ }^{33}$ J. A. N. Gonçales, J. J. Barroso, and G. M. Sandonato, Diamond Relat. Mater. 13, 60 (2004)

${ }^{34}$ G. H. Jansen, Coulomb Interactions in Particle Beams, Advances in Elec- tronics and Electron Physics, Suppl. 21 (Academic, Boston, 1990).

${ }^{35}$ R. J. Umstattd, C. G. Carr, C. L. Frenzen, J. W. Lugisland, and Y. Y. Lau, Am. J. Phys. 73, 160 (2005).

${ }^{36}$ R. G. Forbes and G. L. R. Mair, in Handbook of Charged Particle Optics, 2nd ed., edited by J. Orloff (CRC, Boca Raton, 2008), Chap. 2.

${ }^{37}$ See http://www.mebs.co.uk/Software\%20Demos\%20Book.pdf, valid August 2008.

${ }^{38}$ D. R. Kingham and L. W. Swanson, Appl. Phys. 34, 124 (1984).

${ }^{39}$ R. G. Forbes and N. N. Ljepojevic, Surf. Sci. 246, 113 (1991).

${ }^{40}$ T. Radlicka and B. Lencová, Ultramicroscopy 108, 445 (2008).

${ }^{41}$ P. D. Prewett and G. L. R. Mair, Focused Ion Beams from Liquid Metal Ion Sources (Research Studies, Taunton, UK, 1991).

${ }^{42}$ J. Orloff, L. Swanson, and M. Utlaut, High Resolution Focused Ion Beams: FIB and its Applications (Kluwer, Dordrecht/Plenum, New York, 2003).

${ }^{43}$ F. G. Rudenauer, Surf. Interface Anal. 39, 116 (2007).

${ }^{44}$ C. A. Spindt, I. Brodie, C. E. Holland, and P. R. Schwoebel, in Vacuum Microelectronics, edited by W. Zhu (Wiley, New York, 2001), Chap. 4.

${ }^{45} \mathrm{~N}$. de Jonge, Y. Lamy, K. Schoots, and T. H. Oosterkamp, Nature (London) 420, 393 (2002).

${ }^{46}$ E. L. Murphy and R. H. Good, Phys. Rev. 102, 1464 (1956).

${ }^{47}$ R. G. Forbes and J. H. B. Deane, Proc. R. Soc. London, Ser. A 463, 2907 (2007).

${ }^{48}$ T. J. Lewis, Proc. Phys. Soc. London, Sect. B 68, 938 (1955).

${ }^{49}$ D. Nagy and P. H. Cutler, Phys. Lett. 10, 263 (1964).

${ }^{50}$ J. W. Luginsland, Y. Y. Lau, R. J. Umsttattd, and J. J. Wattous, Phys. Plasmas 9, 2371 (2002)

${ }^{51}$ W. S. Koh and L. K. Ang, Appl. Phys. Lett. 89, 183107 (2006).

${ }^{52}$ J. Norem, Z. Insepov, and I. Konkashbaev, Nucl. Instrum. Methods Phys. Res. A 537, 510 (2005).

${ }^{53}$ R. G. Forbes, Technical Digest of the 20th International Vacuum Nanoelectronics Conference, edited by H. H. Busta, C. A. Spindt, C. E. Hunt, I. Brodie, and E. H. Edwards (IEEE, Piscataway, NJ, 2007), p. 147.

${ }^{54}$ The term "system geometry" includes both the emitter itself and its electrical surroundings.

${ }^{55}$ C. Herring and M. H. Nichols, Rev. Mod. Phys. 21, 185 (1949).

${ }^{56}$ International Standard, ISO 31-0: Quantities and Units: Part 0: General Principles (International Organization for Standardization, Geneva, Switzerland, 1992).

${ }^{57}$ Also known as the Child-Langmuir law.

${ }^{58}$ R. G. Forbes, J. Vac. Sci. Technol. B 26, 788 (2008).

${ }^{59}$ R. G. Forbes, J. Appl. Phys. 103, 114911 (2008).

${ }^{60}$ G. A. Mesyats and I. V. Uimanov, IEEE Trans. Dielectr. Electr. Insul. 13, 105 (2006).

${ }^{61}$ A. Kiejna and K. F. Wojciechowski, Metal Surface Electron Physics (Pergamon, Oxford, 1996).

${ }^{62}$ K. L. Jensen, J. Appl. Phys. 85, 2667 (1999).

${ }^{63}$ K. L. Jensen, J. Appl. Phys. 102, 024911 (2007).

${ }^{64}$ W. P. Dyke and J. K. Trolan, Phys. Rev. 89, 799 (1953).

${ }^{65}$ F. R. Abbott and J. E. Henderson, Phys. Rev. 56, 113 (1939).

${ }^{66}$ G. N. Fursey, Field Emission in Vacuum Microelectronics (Kluwer, Dordrecht/Plenum, New York, 2005).

${ }^{67}$ G. A. Mesyats, Explosive Electron Emission (URO, Ekaterinburg, 1998).

${ }^{68}$ See http://mathworld.wolfram.com/CubicFormula.html, valid April 2008, and citations therein.

${ }^{69}$ R. G. Forbes, J. Vac. Sci. Technol. B 17, 534 (1999). 